\title{
Quadrupole-octupole coupling and the onset of octupole deformation in actinides
}

\author{
K. Nomura, ${ }^{1,}$ 米 R. Rodríguez-Guzmán, ${ }^{2}$ L. M. Robledo, ${ }^{3,4}$ and J. E. García-Ramos ${ }^{5,6}$ \\ ${ }^{1}$ Department of Physics, Faculty of Science, University of Zagreb, HR-10000, Croatia \\ ${ }^{2}$ Physics Department, Kuwait University, 13060 Kuwait, Kuwait \\ ${ }^{3}$ Departamento de Física Teórica and CIAFF, Universidad Autónoma de Madrid, E-28049 Madrid, Spain \\ ${ }^{4}$ Center for Computational Simulation, Universidad Politécnica de Madrid, \\ Campus de Montegancedo, Bohadilla del Monte, E-28660-Madrid, Spain \\ ${ }^{5}$ Departamento de Ciencias Integradas y Centro de Estudios Avanzados en Fúsica, \\ Matemática y Computación, Universidad de Huelva, E-21071 Huelva, Spain \\ ${ }^{6}$ Instituto Carlos I de Física Teórica y Computacional, \\ Universidad de Granada, Fuentenueva s/n, 18071 Granada, Spain
}

(Dated: April 14, 2021)

\begin{abstract}
The evolution of quadrupole and octupole collectivity and their coupling is investigated in a series of even-even isotopes of the actinide $\mathrm{Ra}, \mathrm{Th}, \mathrm{U}, \mathrm{Pu}, \mathrm{Cm}$, and $\mathrm{Cf}$ with neutron number in the interval $130 \leqslant N \leqslant 150$. The Hartree-Fock-Bogoliubov approximation, based on the parametrization D1M of the Gogny energy density functional, is employed to generate potential energy surfaces depending upon the axially-symmetric quadrupole and octupole shape degrees of freedom. The mean-field energy surface is then mapped onto the expectation value of the $s d f$ interacting-bosonmodel Hamiltonian in the boson condensate state as to determine the strength parameters of the boson Hamiltonian. Spectroscopic properties related to the octupole degree of freedom are produced by diagonalizing the mapped Hamiltonian. Calculated low-energy negative-parity spectra, $B\left(E 3 ; 3_{1}^{-} \rightarrow 0_{1}^{+}\right)$reduced transition rates, and effective octupole deformation suggest that the transition from nearly spherical to stable octupole-deformed, and to octupole vibrational states occurs systematically in the actinide region.
\end{abstract}

\section{INTRODUCTION}

Over the decades, octupole deformation in nuclei and the related spectroscopy of negative-parity collective states has been an active research field in low-energy nuclear physics 1, 2. It is well known that the ground state of most medium-mass and heavy nuclei is reflection symmetric and therefore the dominant intrinsic deformation is of quadrupole character. On the other hand, there are a handful of nuclear systems where reflection symmetry is broken, giving rise to an octupole-deformed ground state. The octupole shape is expected to be present in those mass regions corresponding to protons $Z$ and neutron numbers $N$ close to $34,56,88$, and 134 [1,2]. Observables characteristic of the ground state static octupole deformation are low-lying negative-parity bands, which form well deformed quadrupole deformed nuclei and approximate alternating-parity doublet with the ground-state positive-parity band, and enhanced electric dipole and octupole transition rates. Fingerprints of stable octupole shapes have been found experimentally in light actinides $\left({ }^{220} \mathrm{Rn},{ }^{224} \mathrm{Ra}\right.$ and ${ }^{222,228} \mathrm{Ra}$ [3, 4] and $\left.{ }^{228} \mathrm{Th}[5]\right)$ and lanthanides $\left({ }^{144,146} \mathrm{Ba}[6\right.$, [] $)$. Within this context, numerous theoretical investigations have been made to predict, and support evidence of octupole deformation by means of various theoretical models: macroscopic-microscopic models 8 10, self-consistent mean-field (SCMF) methods based on the nuclear density functional theory $11-$ 38, interacting boson model (IBM) 39 45], geometrical

\footnotetext{
* knomura@phy.hr
}

collective models [46 48, and cluster models [49, 50].

Octupole collective excitations in the light actinide nuclei with $Z \approx 88$ and $N \approx 134$ have been extensively studied both experimentally and theoretically. However, spectroscopic data for those actinide nuclei heavier than Th, such as $\mathrm{Pu}, \mathrm{Cm}$, and $\mathrm{Cf}$ isotopes, are scarce, especially in the neutron-deficient side of the nuclear chart with $N \approx 134$. This is because these isotopes are close to the proton drip-line and have not been accessible so far by experiments. It is, nevertheless, worth to explore theoretically whether the robustness of the neutron octupole magic number $N=134$ in actinides holds when one departs from $Z \approx 88$ towards the proton drip-line.

In the present work, we employ the EDF-to-IBM mapping procedure 51] for a theoretical calculation of the properties of octupole collective excitations. This procedure involves two main steps: first, for each nucleus a potential energy surface (PES) depending upon the axially-symmetric quadrupole $\beta_{2}$ and octupole $\beta_{3}$ shape degrees of freedom is computed within the constrained SCMF method with a choice of an universal energy density functional and pairing force. In a second step, the PES is mapped onto the expectation value of the bosonic Hamiltonian in the condensate state of the monopole $s$, quadrupole $d$, and octupole $f$ bosons in order to fix some of the model's parameters [39, 52. Subsequent diagonalization of the resulting boson Hamiltonian yields excitation energy spectra and transition strengths. The mapping procedure has been initially implemented [42, 43] in the study of spectroscopic properties of reflection-asymmetric $\mathrm{Ba}, \mathrm{Sm}, \mathrm{Ra}$, and Th nuclei using the relativistic density-dependent point-coupling 
(DD-PC1) EDF 53 to generate the microscopic PES. More recently, updated calculations have been performed [45] in the ${ }^{218-238} \mathrm{Ra}$ and ${ }^{220-240} \mathrm{Th}$ isotopic chains within the mapped IBM framework based on the Gogny D1M EDF [54. In those studies, consistent with the empirical trend, we have identified a most pronounced octupolarity around the neutron number $N=134$ in the systematic of calculated physical observables.

In view of the renewed interest in a global search for the static octupole deformation beyond $\mathrm{Ra}$ and Th isotopes, here we extend the analysis of 45 to heavier actinide nuclei ${ }^{222-242} \mathrm{U},{ }^{224-244} \mathrm{Pu},{ }^{226-246} \mathrm{Cm}$, and ${ }^{228-248} \mathrm{Cf}$, and verify whether the octupole-related shape phase transition generally occurs in the actinide region, that is, the onset of stable octupole deformation at $N \approx 134$ and octupole softness starting from $N \approx 138$. With the present study we also aim to assess the performance of the EDFbased IBM approach in the global description of octupole collective states in the actinide region. The results further include quantitative predictions on spectroscopy in proton-rich actinides that have not been explored so far by experiment. This has not been possible in the previous IBM calculations, since they are mostly fit to known experimental data. Hence, the present work points not only to an alternative EDF-based approach to the detailed spectroscopy of a large number of actinide nuclei, but it is a first implementation of the IBM framework in the octupole-related spectroscopic studies on proton-rich heavy actinides, that is based on the microscopic EDF.

It should be noted that interplay between quadrupole and octupole degrees of freedom in the low-lying negative parity collective states of $\mathrm{U}, \mathrm{Pu}, \mathrm{Cm}$, and $\mathrm{Cf}$ nuclei has also been studied within the framework of the generator coordinate method (GCM) [55 57] using the Gogny D1M EDF 38. The GCM calculation is, however, quite time consuming especially for heavy systems or when the number of collective coordinates increases. Computational complexity also prevents to have access to high spin states like, for instance, the members of alternating parity rotational bands. In Refs. 33, 34, a large number of heavy and superheavy nuclei up to No isotopes with mass $A \approx 300$ have been analyzed by solving quadrupoleoctupole collective Hamiltonian, with parameters specified by the relativistic EDF calculations. Detailed spectroscopy of stable $\mathrm{U}$ and $\mathrm{Pu}$ nuclei has been explored within a purely phenomenological spdf-IBM framework in Refs. [41, 58.

The paper is organized as follows. The mapping procedure used to obtain the IBM Hamiltonian is illustrated in Sec. II] The results of our analysis are discussed in Sec. III including the Gogny-D1M quadrupole-octupole SCMF-PESs, i.e., the microscopic building blocks of the calculations, mapped IBM-PESs, low-energy excitation spectra, transition properties, and the effective $\beta_{2}$ and $\beta_{3}$ deformation parameters. Finally, Sec. IV] is devoted to the concluding remarks and work perspectives.

\section{THEORETICAL METHOD}

To obtain the quadrupole-octupole SCMF-PESs, the HFB equation has been solved with constrains on the axially symmetric quadrupole $\hat{Q}_{20}$ and octupole $\hat{Q}_{30}$ operators [27, 38]. The mean values $\left\langle\Phi_{\mathrm{HFB}}\left|\hat{Q}_{20}\right| \Phi_{\mathrm{HFB}}\right\rangle=Q_{20}$ and $\left\langle\Phi_{\mathrm{HFB}}\left|\hat{Q}_{30}\right| \Phi_{\mathrm{HFB}}\right\rangle=Q_{30}$ define the quadrupole and octupole deformation parameters $\beta_{\lambda}(\lambda=2,3)$, i.e., $\beta_{\lambda}=\sqrt{4 \pi(2 \lambda+1)} Q_{\lambda 0} /\left(3 R_{0}^{\lambda} A\right)$, with $R_{0}=1.2 A^{1 / 3} \mathrm{fm}$. The constrained calculations provide a set of HFB states $\left|\Phi_{\mathrm{HFB}}\left(\beta_{2}, \beta_{3}\right)\right\rangle$ labeled by their static deformation parameters $\beta_{2}$ and $\beta_{3}$. The HFB energies $E_{\mathrm{HFB}}\left(\beta_{2}, \beta_{3}\right)$ associated with those HFB states define the so-called SCMF-PESs used in this work. As the HFB energies satisfy the property $E_{\mathrm{HFB}}\left(\beta_{2}, \beta_{3}\right)=E_{\mathrm{HFB}}\left(\beta_{2},-\beta_{3}\right)$ only positive $\beta_{3}$ values are considered when plotting the SCMF-PESs.

Excitation energies and transition probabilities of the quadrupole and octupole collective states are computed by diagonalizing the IBM Hamiltonian that is determined with microscopic input from the Gogny-HFB SCMF calculation - see below. For computing negative-parity states, we consider the $J=0^{+}(s), 2^{+}(d)$, and $J=3^{-}$ $(f)$ bosons as building blocks of the IBM. The total number of bosons $n=n_{s}+n_{d}+n_{f}$ is conserved for a given nucleus, and is equal to half the number of valence nucleons. The doubly-magic nucleus ${ }^{208} \mathrm{~Pb}$ is taken here as the inert core, and thus $n=(A-208) / 2$ for a nucleus with mass $A$. We adopt the $s d f$-IBM Hamiltonian [45]:

$$
\hat{H}_{\mathrm{IBM}}=\epsilon_{d} \hat{n}_{d}+\epsilon_{f} \hat{n}_{f}+\kappa_{2} \hat{Q}_{2} \cdot \hat{Q}_{2}+\rho \hat{L} \cdot \hat{L}+\kappa_{3} \hat{Q}_{3} \cdot \hat{Q}_{3} .
$$

The first (second) term represents the number operator for the $d(f)$ bosons with $\epsilon_{d}\left(\epsilon_{f}\right)$ being the single $d(f)$ boson energy relative to the $s$ boson one. The third, fourth, and fifth terms represent the quadrupole-quadrupole interaction, the rotational term, and the octupole-octupole interaction, respectively. The quadrupole $\hat{Q}_{2}$, the angular momentum $\hat{L}$, and the octupole $\hat{Q}_{3}$ operators are expressed as

$$
\begin{aligned}
& \hat{Q}_{2}=s^{\dagger} \tilde{d}+d^{\dagger} \tilde{s}+\chi_{d}\left[d^{\dagger} \times \tilde{d}\right]^{(2)}+\chi_{f}\left[f^{\dagger} \times \tilde{f}\right]^{(2)} \\
& \hat{L}=\sqrt{10}\left[d^{\dagger} \times \tilde{d}\right]^{(1)}+\sqrt{28}\left[f^{\dagger} \times \tilde{f}\right]^{(1)} \\
& \hat{Q}_{3}=s^{\dagger} \tilde{f}+f^{\dagger} \tilde{s}+\chi_{3}\left[d^{\dagger} \times \tilde{f}+f^{\dagger} \times \tilde{d}\right]^{(3)}
\end{aligned}
$$

Note that the term proportional to $\left(d^{\dagger} \tilde{d}\right)^{(1)} \cdot\left(f^{\dagger} \tilde{f}\right)^{(1)}$ in the $\hat{L} \cdot \hat{L}$ term has been neglected [45]. The parameters $\epsilon_{d}, \epsilon_{f}, \kappa_{2}, \rho, \chi_{d}, \chi_{f}, \kappa_{3}$, and $\chi_{3}$ of the $s d f$ IBM Hamiltonian are determined, for each nucleus, in such a way [44, 45] that the expectation value of the $s d f$-IBM Hamiltonian in the boson condensate state, $E_{\mathrm{IBM}}\left(\beta_{2}, \beta_{3}\right)=\left\langle\phi\left(\beta_{2}, \beta_{3}\right)\left|\hat{H}_{\mathrm{IBM}}\right| \phi\left(\beta_{2}, \beta_{3}\right)\right\rangle$, reproduces the Gogny-HFB SCMF PES $E_{\mathrm{HFB}}\left(\beta_{2}, \beta_{3}\right)$ in the neighborhood of the global minimum. The boson condensate wave function is given by [59]:

$$
\left|\phi\left(\beta_{2}, \beta_{3}\right)\right\rangle=(n !)^{-1 / 2}\left(s^{\dagger}+\bar{\beta}_{2} d_{0}^{\dagger}+\bar{\beta}_{3} f_{0}^{\dagger}\right)^{n}|0\rangle,
$$


where $|0\rangle_{-}$denotes the inert core, i.e., ${ }^{208} \mathrm{~Pb}$. The amplitudes $\bar{\beta}_{2}$ and $\bar{\beta}_{3}$ entering the definition of the boson condensate wave function are proportional to the deformation parameters $\beta_{2}$ and $\beta_{3}$ of the fermionic space, $\bar{\beta}_{2}=C_{2} \beta_{2}$ and $\bar{\beta}_{3}=C_{3} \beta_{3}$, 43, 44, 59, with dimensionless proportionality constants $C_{2}$ and $C_{3}$. Their values are also determined by the mapping procedure so that the location of the global minimum in the SCMF-PES, denoted by $\beta_{2, \text { min }}$ and $\beta_{3, \min }$, is reproduced. A more detailed description of the whole procedure can be found in Ref. 45]. As for the analytical expression of the IBMPES $E_{\mathrm{IBM}}\left(\beta_{2}, \beta_{3}\right)$, we refer the reader to Ref. [44].

For the numerical diagonalization of the Hamiltonian $\hat{H}_{\text {IBM }}$ (1), the computer code ARBModEL 60] has been used.
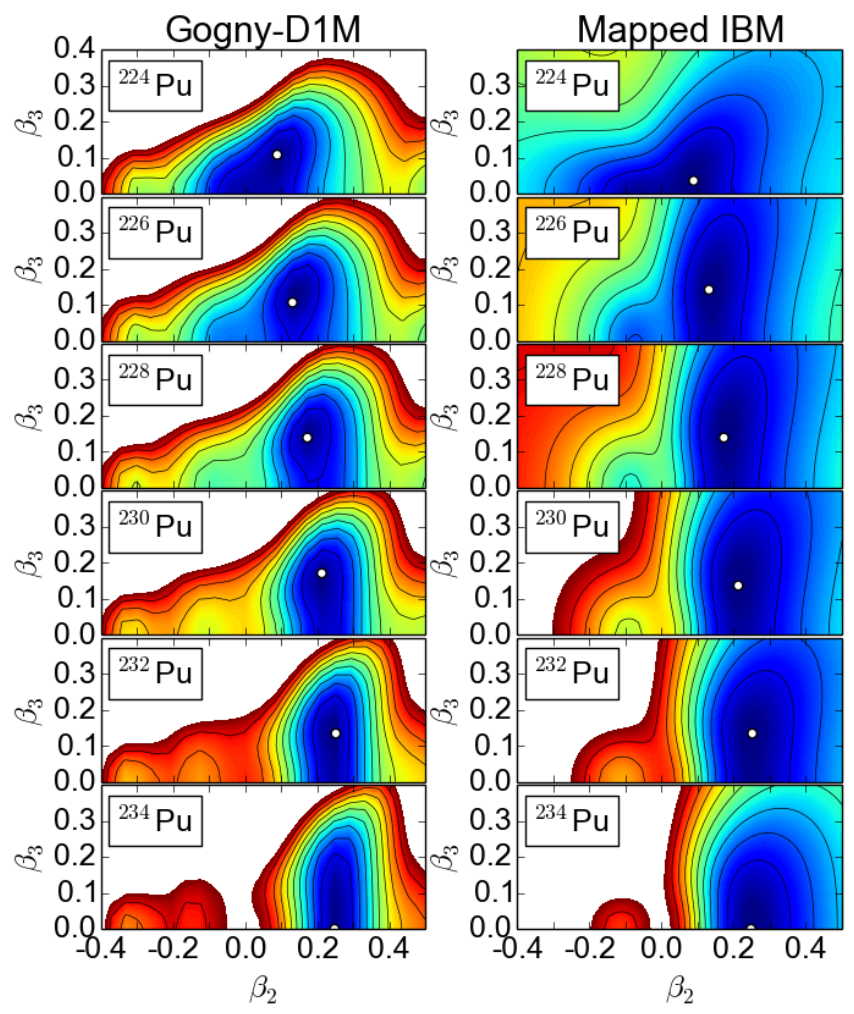

FIG. 1. SCMF-PESs computed with the Gogny-D1M EDF for the nuclei ${ }^{224-234} \mathrm{Pu}$ (left), and the corresponding mapped $s d f$-IBM PESs (right). The color code indicates the total HFB and IBM energies in $\mathrm{MeV}$ units, plotted up to $10 \mathrm{MeV}$ with respect to the global minimum. Energy difference between neighboring contours is $1 \mathrm{MeV}$. For each nucleus, the global minimum is indicated by an open circle.
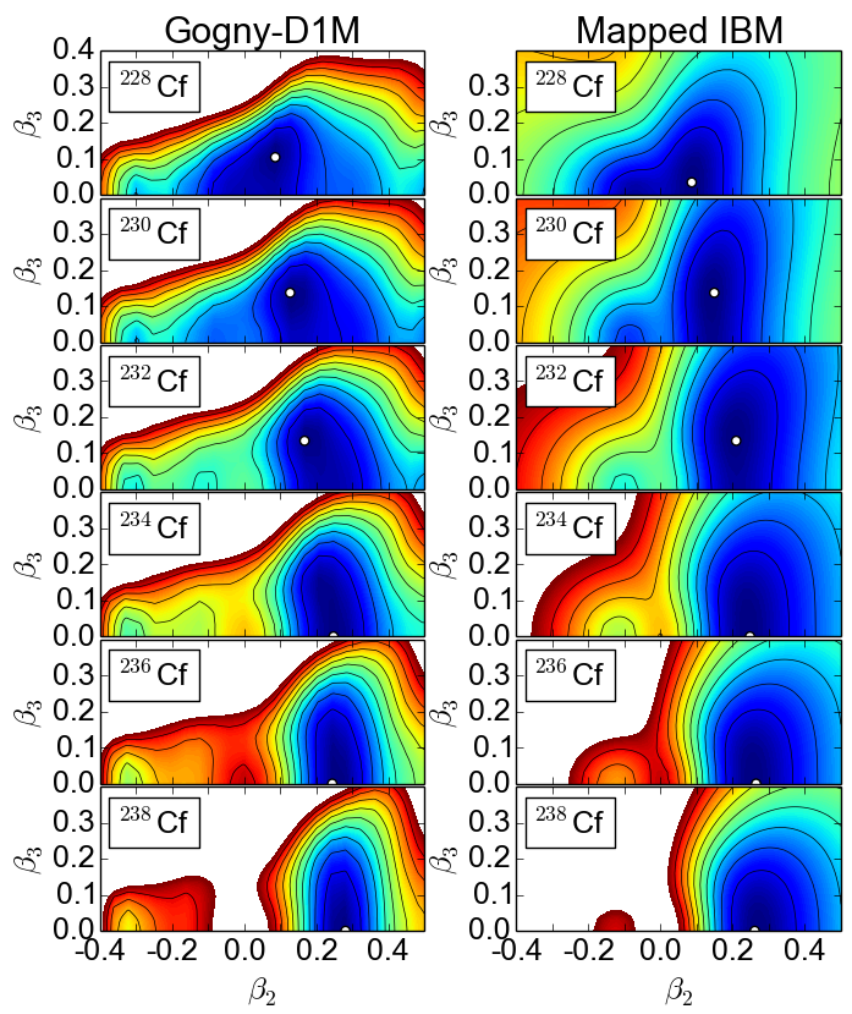

FIG. 2. Same as for Fig. 1] but for ${ }^{248-238} \mathrm{Cf}$.

\section{RESULTS AND DISCUSSIONS}

\section{A. Gogny-D1M SCMF-PESs}

As representative cases, the Gogny-D1M SCMF-PESs for ${ }^{224-234} \mathrm{Pu}$ and ${ }^{228-238} \mathrm{Cf}$ are depicted in the left columns of Figs. 1 and 2 , respectively. In most of the $\mathrm{Pu}$ isotopes, a non-zero $\beta_{3}$ minimum $\beta_{3, \min }$ is found in the interval of $0.1 \leqslant \beta_{3} \leqslant 0.2$. The most pronounced octupole minimum is obtained at $N=134\left({ }^{228} \mathrm{Pu}\right)$ around $\beta_{3, \min } \approx 0.17$. For Cf nuclei in Fig. 2, only three isotopes are octupole deformed with $\beta_{3 \text {,min }}<0.15$. Note that for those isotopes with $N>140$, the potential energy becomes softer in $\beta_{3}$ and no octupole deformation is found.

The systematic of the SCMF-PESs for U and Cm isotopes is similar to the one for $\mathrm{Pu}$ and $\mathrm{Cf}$ isotopes, and the PESs for Ra and Th can be found in Ref. 45. It is also worth mentioning that the Gogny-D1M SCMF-PESs used in this paper are very similar to those obtained in Ref. 26] using the Gogny-D1S 61] and D1N 62], and the Barcelona-Catania-Paris (BCP) 23] EDFs. Recent comparisons of several non-relativistic Skyrme and relativistic EDFs in a survey of octupole correlations can be found in Refs. 37] and [31, respectively. In those references, it is noticed that for most of the adopted EDFs pronounced octupole mean-field minimum occur around 

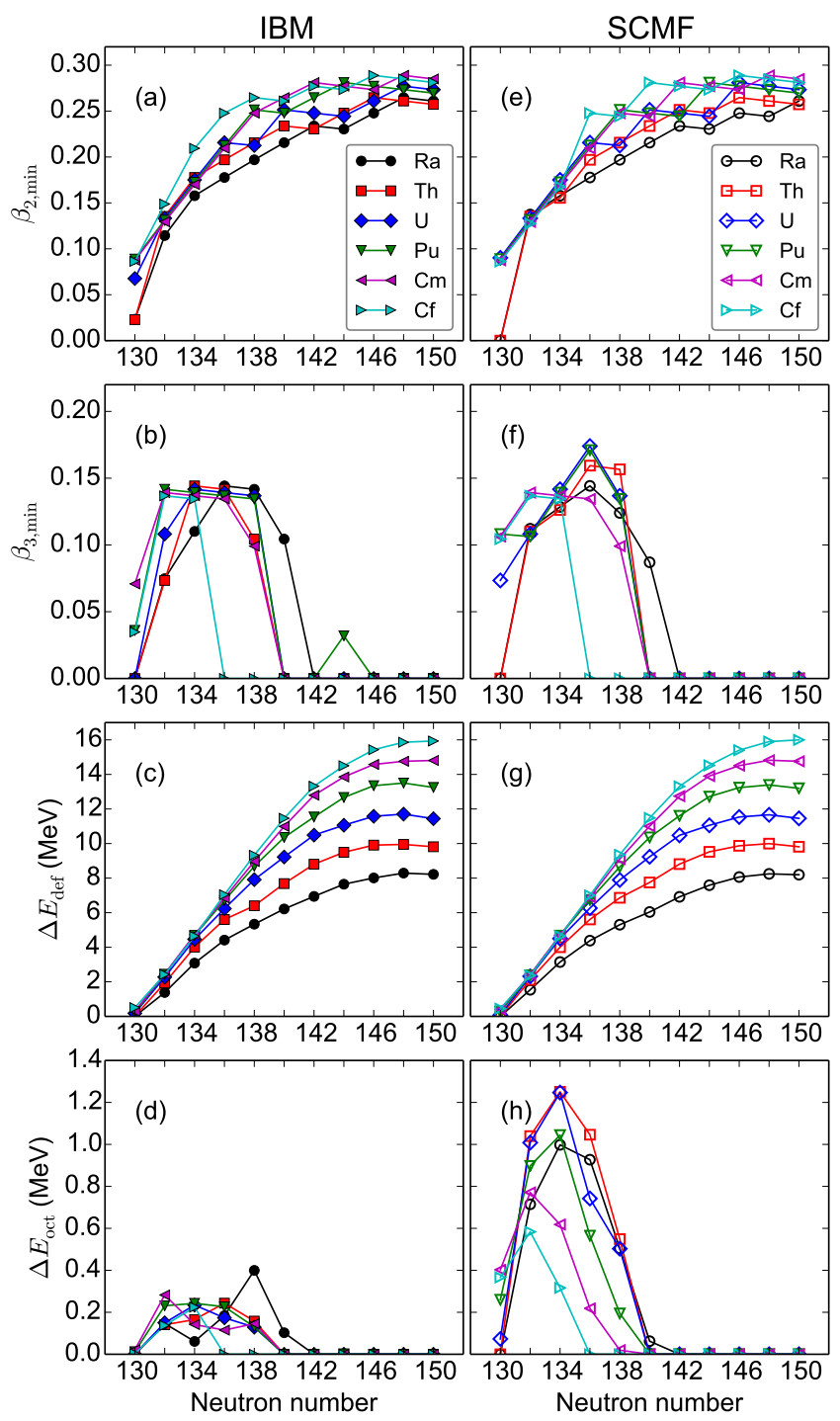

FIG. 3. The $\beta_{2, \text { min }}$ and $\beta_{3, \text { min }}$ values, corresponding to the ground state minimum are plotted as a function of neutron number in panels (a) and (b), respectively. In panel (c) the deformation energy $\Delta E_{\text {def }}$, defined as the energy difference between the global minimum and the spherical configuration is plotted as a function of neutron number. The octupole deformation energy $\Delta E_{\text {oct }}$, defined as the energy difference between the global minimum and the quadrupole deformed minimum along the $\beta_{3}=0$ axis, for the mapped IBM-PESs is plotted in panel (d). The corresponding quantities for the SCMF-PESs are also plotted in the right hand side panels from (e) to (h). The results for the Ra and Th nuclei have been taken from Ref. [45]. See the main text for definitions of the above quantities.

$N=134$
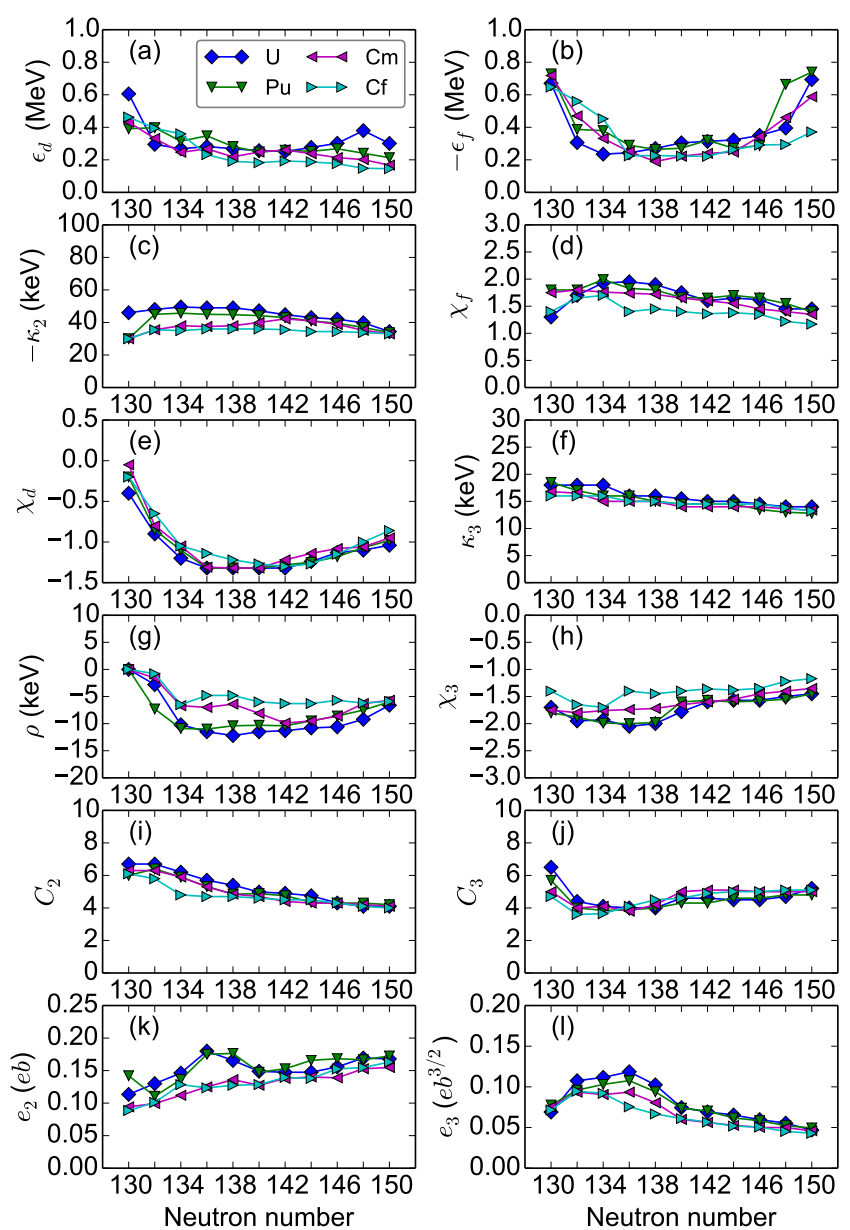

FIG. 4. The strength parameters $\epsilon_{d}, \epsilon_{f}, \kappa_{2}, \chi_{f}, \chi_{d}, \kappa_{3}, \rho$ and $\chi_{3}$ of the $s d f$-IBM Hamiltonian Eq. (1) are plotted as a function of neutron number in panels from (a) to (h) for the four isotopic chains considered. The coefficients $C_{2}$ and $C_{3}$ connecting the IBM and microscopic quadrupole and octupole deformation parameters are plotted in panels (i) and (j). Finally, the boson effective charges for the quadrupole $e_{2}$ and octupole $e_{3}$ transitions are plotted as a function of the neutron number in panels $(\mathrm{k})$ and $(\mathrm{l})$, respectively. The parameters for $\mathrm{Ra}$ and $\mathrm{Th}$ isotopes can be found in Ref. [45].

\section{B. Mapped IBM-PESs}

The corresponding IBM-PESs are drawn on the righthand sides of Figs. 1 and 2 2 One can clearly observe the similarities between the SCMF and IBM PESs: the topography of the IBM-PES changes with the neutron number in a similar way as the SCMF-PES, from nearly spherical configurations $(N \approx 130)$ to pronounced octupole deformation $(N \approx 134)$ continuing with $\beta_{3}$-soft $(N \approx 138)$. The IBM-PESs are generally softer than the SCMF-PESs. This is related to the more restricted configuration space of the IBM as compared to the one of the SCMF model.

In Fig 3 we show, as a function of neutron number, 
the values of several quantities characterizing the selfconsistent minima found in the IBM (left panels) and SCMF PES (right panels). On panels (a) and (b) the values of the quadrupole and octupole deformation parameters of the absolute minimum $\beta_{2 \text {,min }}$ and $\beta_{3, \text { min }}$ are plotted. On panel (c) the quadrupole deformation energy $\Delta E_{\text {def }}$ defined as the energy difference between the energy at the global minimum and at the spherical point:

$$
\Delta E_{\text {def }}=E\left(\beta_{2, \min }, \beta_{3, \min }\right)-E(0,0),
$$

is shown, where $E\left(\beta_{2}, \beta_{3}\right)$ refers either to the SCMFPES or the IBM-PES. Finally, the octupole deformation energies $\Delta E_{\text {oct }}$, defined as the energy difference between the energy at the global minimum and the local minimum on the $\beta_{3}=0$ axis, i.e.

$$
\Delta E_{\text {oct }}=E\left(\beta_{2, \min }, \beta_{3, \min }\right)-E\left(\beta_{2, \min }^{\prime}, 0\right),
$$

is shown in panel (d). In the above expression $\beta_{2, \text { min }}^{\prime}$ stands for the $\beta_{2}$ deformation parameter corresponding to the local minimum on the $\beta_{3}=0$ axis. The corresponding quantities calculated with the SCMF-PESs $E_{\mathrm{HFB}}\left(\beta_{2}, \beta_{3}\right)$ are included in panels from (e) to (h) in the plot. The systematic as a function of neutron number of most of these quantities is basically the same when looking at the SCMF and IBM PESs.

Only one exception can be seen in the quantity $\Delta E_{\text {oct }}$ shown in Fig. 3 panel (d): the IBM values are a factor between two to five lower than the SCMF ones. This discrepancy reflects the fact that the IBM-PESs are much softer in $\beta_{3}$ direction than the SCMF-PESs: the latter displays a much steeper potential in $\beta_{3}$ than the former (see also Figs. 1 and 2). It is partly attributed to the fact that the analytical form of the IBM-PES [44] is so restricted, comprising only limited number and species of bosons, that it is not able to reproduce in full detail the topology of the SCMF-PES, which is much steeper in $\beta_{3}$ direction, but only the overall topology of the SCMF-PES typically up to a few $\mathrm{MeV}$ from the minimum. However, the SCMF solutions within this energy range are most relevant to low-lying states. In addition, the fact that the IBM-PESs are considerably $\beta_{3}$ soft as compared to the SCMF ones presents a general feature of the IBM framework, but is considered to be of minor relevance to reproducing spectroscopic properties of low-energy yrast state and thus does not alter the main conclusions.

\section{Derived IBM parameters}

In Fig. 4 we display the IBM parameters obtained for the considered $\mathrm{U}, \mathrm{Pu}, \mathrm{Cm}$, and $\mathrm{Cf}$ nuclei as functions of the neutron number $N$. Most of the derived parameters appear to stay nearly constant with $N$, and their values also do not significantly differ between different isotopic chains. This is conceptually very satisfying as it indicates the consistency of the approach and its predictive power. An exception is perhaps the $f$-boson energy $\epsilon_{f}$ depicted in panel (b) of Fig. 4, which exhibits an abrupt structural change between neighboring isotopes. The rapid decrease of the quantity $-\epsilon_{f}$ from $N=130$ to 136 indicates the development of the octupole collectivity.
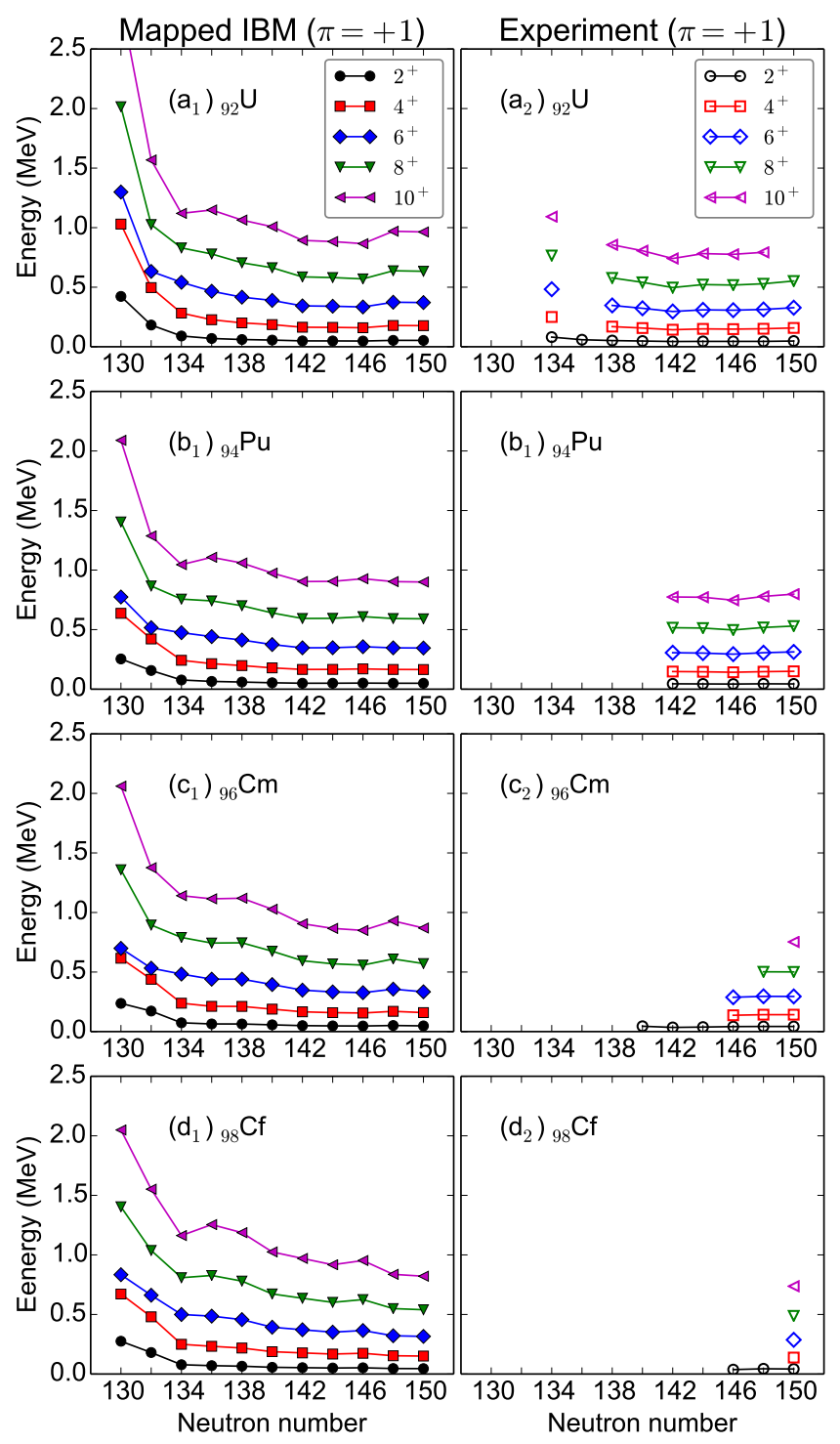

FIG. 5. Low-energy excitation spectra of positive-parity evenspin yrast states of ${ }^{222-242} \mathrm{U},{ }^{224-244} \mathrm{Pu},{ }^{226-246} \mathrm{Cm}$, and ${ }^{228-248} \mathrm{Cf}$ computed by the diagonalization of the mapped $s d f$-IBM Hamiltonian Eq. (1). Experimental data are taken from Ref. 63. Results for Ra and Th isotopes can be found in Ref. 45.

\section{Evolution of low-energy excitation spectra}

The excitation spectra for the low-lying even-spin positive-parity and odd-spin negative-parity yrast states of ${ }^{222-242} \mathrm{U},{ }^{224-244} \mathrm{Pu},{ }^{226-246} \mathrm{Cm}$, and ${ }^{228-248} \mathrm{Cf}$ are depicted in Figs. 5 and 6 as functions of $N$, respectively. 

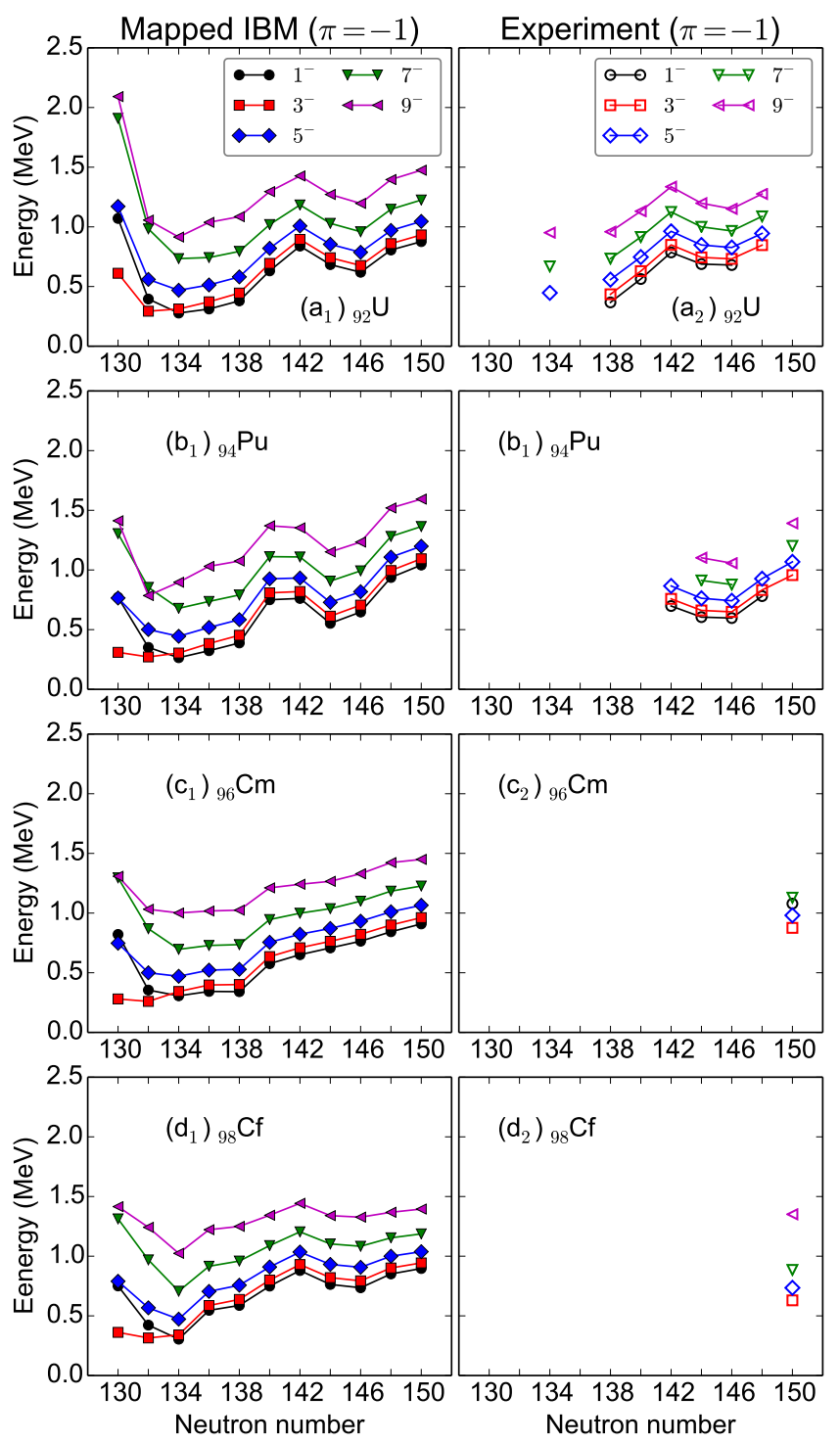

FIG. 6. Same as the caption of Fig. 5 but for the odd-spin negative-parity yrast states.

In Fig. 5, the calculated positive-parity levels in each isotopic chain (panels $\left(\mathrm{a}_{1}\right)$ to $\left(\mathrm{d}_{1}\right)$ ) are seen to decrease from $N=130$ to 134 , as the quadrupole collectivity develops. A typical rotational band structure starts to appear from $N \approx 132$, and there is no significant change in positiveparity states from $N=134$ on. Agreement between the theoretical and experimental (panels from $\left(\mathrm{a}_{2}\right)$ to $\left(\mathrm{d}_{2}\right)$ ) positive-parity levels is remarkable. It should be noted that the predicted level structure of the transitional nuclei with $N=130$ and 132 looks rather irregular: energy levels of the $4_{1}^{+}$and $6_{1}^{+}$are close to each other, at variance with the well-known vibrational or rotational band patterns. As we show in Sec. IIIE, such a irregularity in the calculated levels seems to occur due to strong mixing between positive- and negative-parity boson configurations in the low-spin states of these nuclei.

The systematic behavior of the calculated negativeparity states is depicted in Fig. 6 (panels $\left(a_{1}\right)$ to $\left(d_{1}\right)$ ). The comparison of our results with the available experimental data (panels from $\left(\mathrm{a}_{2}\right)$ to $\left(\mathrm{d}_{2}\right)$ ) is reasonable. For each considered isotopic chain, the predicted negativeparity states exhibit an approximate parabolic behavior centered around $N=134$ which corresponds with the nuclei where the octupole minimum is most pronounced in the SCMF-PESs. From $N=134$ on, the negative-parity level energies keep increasing up to $N=142$, where another parabolic-like behavior sets in the $\mathrm{U}, \mathrm{Pu}$ and $\mathrm{Cf}$ isotopes. The absence of permanent octupole deformation in those isotopes hints to an increasing dominant role of dynamic octupole correlations.
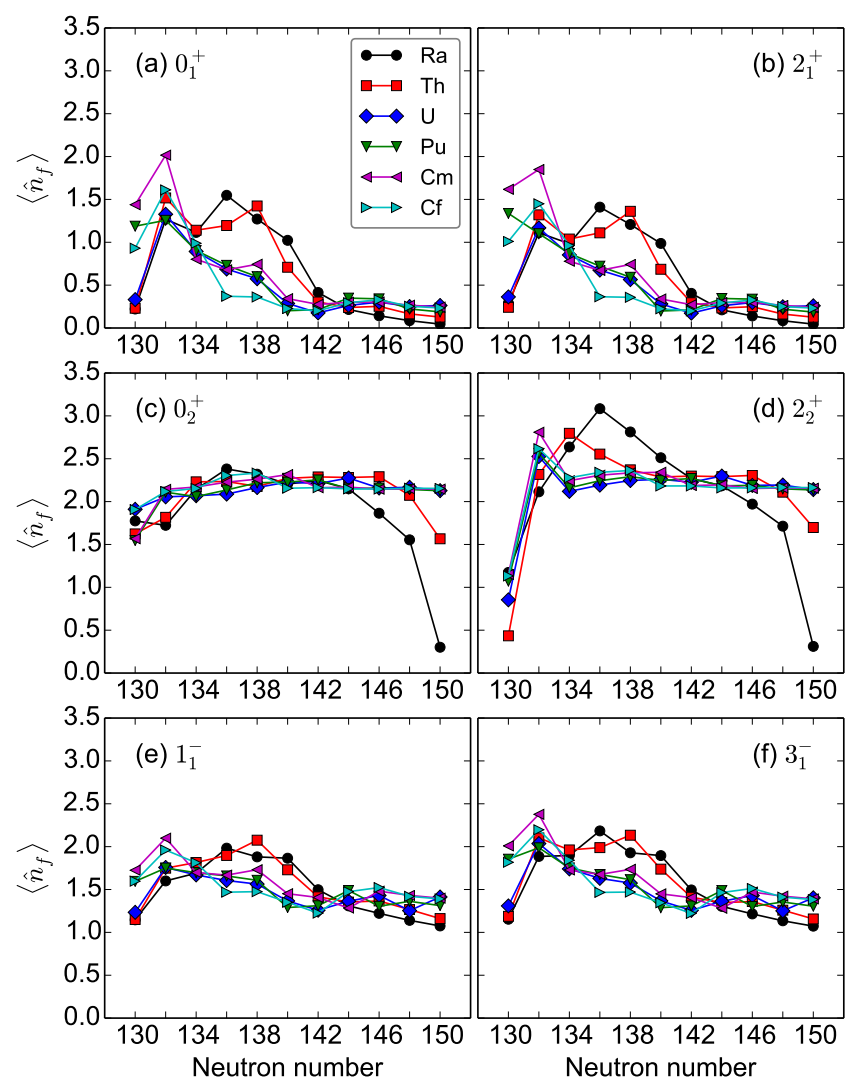

FIG. 7. Expectation values of the $f$-boson number operator $\left\langle\hat{n_{f}}\right\rangle$ in the IBM wave functions of the states $0_{1}^{+}, 2_{1}^{+}, 0_{2}^{+}, 2_{2}^{+}$, $1_{1}^{-}$, and $3_{1}^{-}$of the $\mathrm{Ra}, \mathrm{Th}, \mathrm{U}, \mathrm{Pu}, \mathrm{Cm}$, and $\mathrm{Cf}$ nuclei, plotted as functions of the neutron number. The values for the Ra and $\mathrm{Th}$ isotopes have been taken from [45].

\section{E. $f$-boson contribution to low-lying states}

Next, we discuss the contribution of the $f$ boson to the wave functions of the low-lying positive and negative parity states. Figure 7 displays the expectation values 
of the $f$-boson number operator $\left\langle\hat{n}_{f}\right\rangle$ computed in the IBM wave functions of the states $0_{1}^{+}, 2_{1}^{+}, 0_{2}^{+}, 2_{2}^{+}, 1_{1}^{-}$, and $3_{1}^{-}$. From panels (a) and (b) of Fig. 7, we learn that the expectation value $\left\langle\hat{n}_{f}\right\rangle$ is rather large in the transitional region $132 \leqslant N \leqslant 138$ indicating that the mixing between the $s-d$ and $f$ boson spaces is significant in the ground-state band. There is a marked difference in the results between the $\mathrm{Ra}$ and $\mathrm{Th}$ isotopes and the $\mathrm{U}, \mathrm{Pu}, \mathrm{Cm}$, and $\mathrm{Cf}$ isotopes with the neutron numbers $136 \leqslant N \leqslant 140$ : the $f$-boson contributions to the ground-state bands of the $\mathrm{U}, \mathrm{Pu}, \mathrm{Cm}$, and $\mathrm{Cf}$ isotopes are by a factor two to five smaller than for the Ra and Th nuclei. Thus it follows that, in the present framework, the quadrupole-octupole correlations become weaker as the proton number increases. For $N$ larger than 140, both the $0_{1}^{+}$and $2_{1}^{+}$states are made of the $s$ and $d$ bosons alone, as the expectation values $\left\langle\hat{n}_{f}\right\rangle \approx 0$.

By looking at panels (c) and (d) in Fig. 7. we conclude that the structure of the wave functions of the $0_{2}^{+}$and $2_{2}^{+}$ states corresponds to a double-octupole phonon structure as the expectation value $\left\langle\hat{n}_{f}\right\rangle \approx 2$. Empirical studies have interpreted that low-energy $K=0^{+}$excited bands in the actinide region are partly accounted for by the coupling between double octupole phonons [41, 58]. Both isotopic and isotonic dependencies of these values are not as strong as in the cases of the $0_{1}^{+}$and $2_{1}^{+}$states.

In panels (e) and (f) of Fig. 7 we notice that for the $1_{1}^{-}$and $3_{1}^{-}$states $1.5 \leqslant\left\langle\hat{n}_{f}\right\rangle \leqslant 2.0$ when $N<140$. For larger values of $N,\left\langle\hat{n}_{f}\right\rangle$ gradually decreases to reach the value of one at $N \approx 150$. Therefore, we conclude that more than one $f$ boson is needed for the employed EDFto-IBM mapping procedure to reasonably describe excitation spectra of low-lying negative-parity yrast states in actinide nuclei with $N \leqslant 140$, while for heavier actinides, inclusion of only one $f$ boson seems to suffice.

\section{F. Possible alternating-parity band structure}

To distinguish if the members of rotational bands are octupole-deformed or octupole vibrational states, it is convenient to consider the quantity

$$
\delta E\left(J^{-}\right)=E\left(J^{-}\right)-\frac{E\left((J+1)^{+}\right)+E\left((J-1)^{+}\right)}{2},
$$

where $E\left(J^{-}\right)$and $E\left((J \pm 1)^{+}\right)$represent excitation energies of the odd-spin negative-parity and even-spin positive-parity yrast states, respectively. If the positiveand negative-parity bands share an octupole deformed band head they form an alternating-parity doublet and the quantity $\delta E\left(J^{-}\right)$should be equal to zero. The deviation from the limit $\delta E\left(J^{-}\right)=0$ means that the positive- and negative-parity bands form separate bands, and therefore an octupole vibrational structure emerges. In Fig. 8, the calculated $\delta E\left(J^{-}\right) / E\left(2_{1}^{+}\right)$values for the $J=1_{1}^{-}$to $9_{1}^{-}$states are plotted as functions of $N$. Let us take as examples the results for the $\mathrm{Ra}$ and $\mathrm{Th}$ isotopic chains in panels (a) and (b) of Fig. 8. The ratios
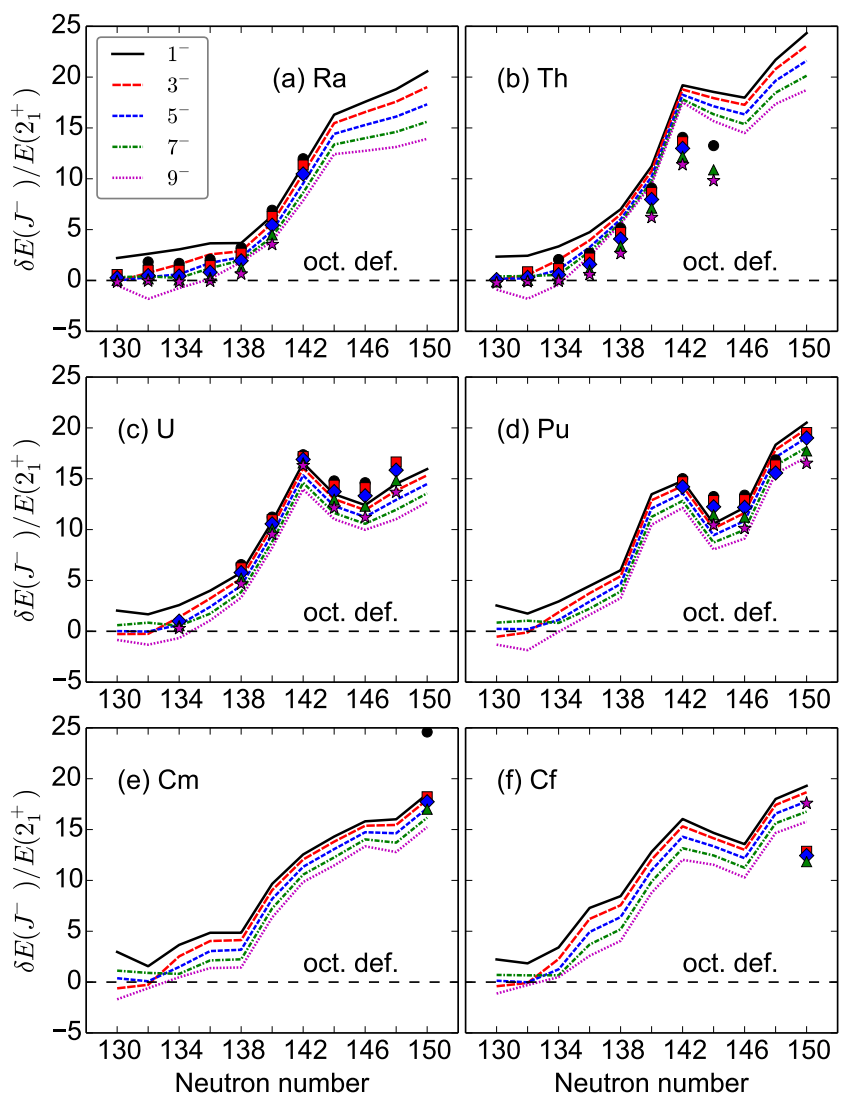

FIG. 8. The energy displacement $\delta E\left(J^{-}\right)$[defined in Eq. 8 $]$], normalized with respect to the excitation energy of the $2_{1}^{+}$ state, is shown as a function of the neutron number. The theoretical values are connected by lines. The corresponding experimental 63. values for the $J^{-}=1^{-}, 3^{-}, 5^{-}, 7^{-}$, and $9^{-}$yrast states are represented by the solid circles, squares, diamond, triangles, and stars, respectively. Results for the Ra and Th nuclei are from 45]. The limit of stable octupole deformation $\delta E\left(J^{-}\right)=0$ is indicated in each panel by a broken horizontal line.

$\delta E\left(J^{-}\right) / E\left(2_{1}^{+}\right)$for each spin are close to zero for a number of Ra and Th isotopes with neutron numbers below $N \approx 138$, i.e., ${ }^{218-226} \mathrm{Ra}$ and ${ }^{220-228} \mathrm{Th}$. For heavier isotopes with $N \geqslant 140, \delta E\left(J^{-}\right) / E\left(2_{1}^{+}\right)$values turn to increase with $N$. Thus the octupole vibrational states characterized by the octupole-soft potential appear. Essentially the same trend is observed for the $\mathrm{U}, \mathrm{Pu}, \mathrm{Cm}$, and $\mathrm{Cf}$ isotopes.

\section{G. Transition rates}

Transition probabilities are computed with the electric dipole, quadrupole and octupole transition operators $\hat{T}^{E \lambda}=e_{\lambda} \hat{Q}_{\lambda}(\lambda=1,2,3)$. As in Ref. [45], the effective charge $e_{\lambda}$ for $\lambda=2$ and 3 depends on the boson number $n$, and is determined so that the IBM's 

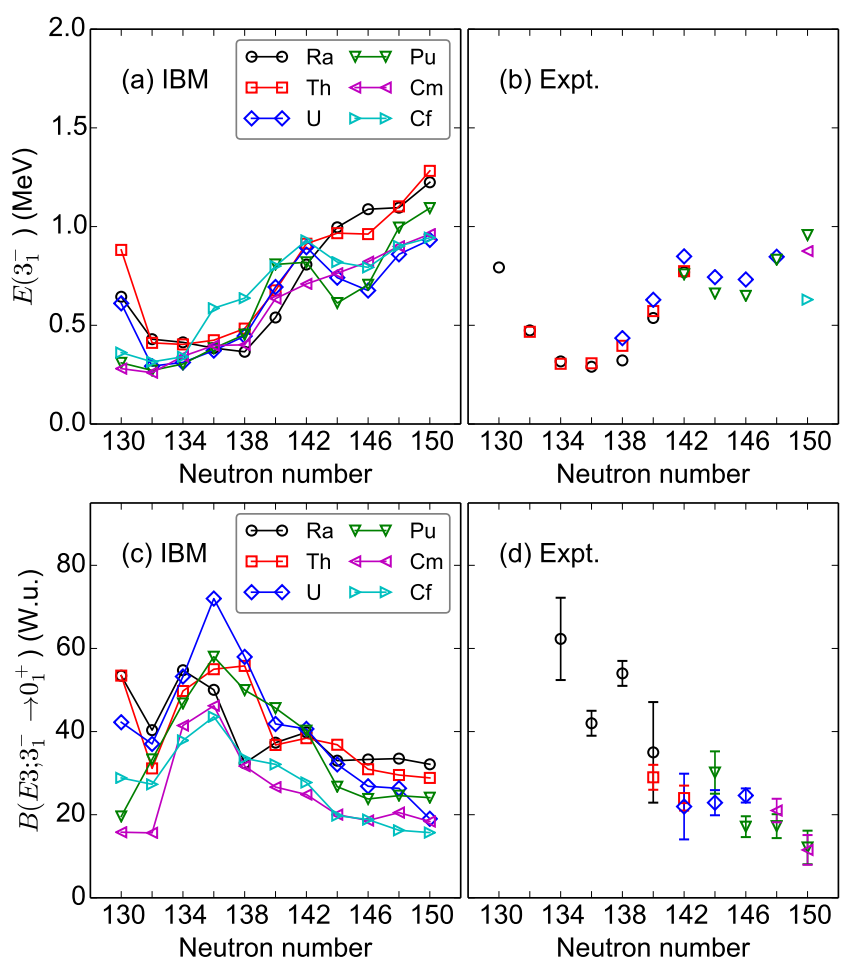

FIG. 9. Evolution of the theoretical and experimental excitation energy of the $3_{1}^{-}$state (panels (a) and (b)), and the $B\left(E 3 ; 3_{1}^{-} \rightarrow 0_{1}^{+}\right)$transition strength in Weisskopf units (panels (c) and (d)) for the Ra, Th, U, Pu, Cm, and Cf isotopes as functions of the neutron number. Theoretical values for the $\mathrm{Ra}$ and Th nuclei are taken from Ref. 45. The experimental data are from Refs. 63, 64]

intrinsic quadrupole (octupole) moments calculated at $\beta_{\lambda}=\beta_{\lambda \text {,min }}$ coincide with the SCMF ones. Formulas used to determine the values of $e_{\lambda}$ can be found in Ref. 45. In the present calculations, a slight modification to the formulas given in [45] has been considered: for the $\mathrm{Cm}$, and $\mathrm{Cf}$ isotopes, an overall factor of the $e_{2}$ charge has been re-scaled so that the experimental $B\left(E 2 ; 2_{1}^{+} \rightarrow 0_{1}^{+}\right)$values for ${ }^{244,246} \mathrm{Cm}$ are reasonably reproduced. The employed $e_{\lambda=2,3}$ values are also shown in panels (k) and (l) of Fig. 4 . The E1 transition operator is defined in 45, and the same value of the E1 charge $e_{1}=0.0277 \mathrm{eb}^{1 / 2}$ is used for all the considered isotopic chains. The $B\left(E 3 ; 3_{1}^{-} \rightarrow 0_{1}^{+}\right)$transition rates as well as the excitation energies of the $3_{1}^{-}$state are depicted as functions of $N$ in Fig. 9. The predicted $B(E 3)$ values show marked peaks at around $N=136$ with a maximum value of $72 \mathrm{~W} . \mathrm{u}$. for ${ }^{228} \mathrm{U}$. This trend is correlated with the behaviors of the calculated and experimental $E\left(3_{1}^{-}\right)$ values plotted in panels (a) and (b) of the same figure: the $B\left(E 3 ; 3_{1}^{-} \rightarrow 0_{1}^{+}\right)$values are inversely proportional to the $E\left(3_{1}^{-}\right)$ones.

In addition, we compare in Fig. 10 the calculated and experimental $B\left(E 2 ; 2_{1}^{+} \rightarrow 0_{1}^{+}\right)$and $B\left(E 1 ; 1_{1}^{-} \rightarrow 0_{1}^{+}\right)$val-
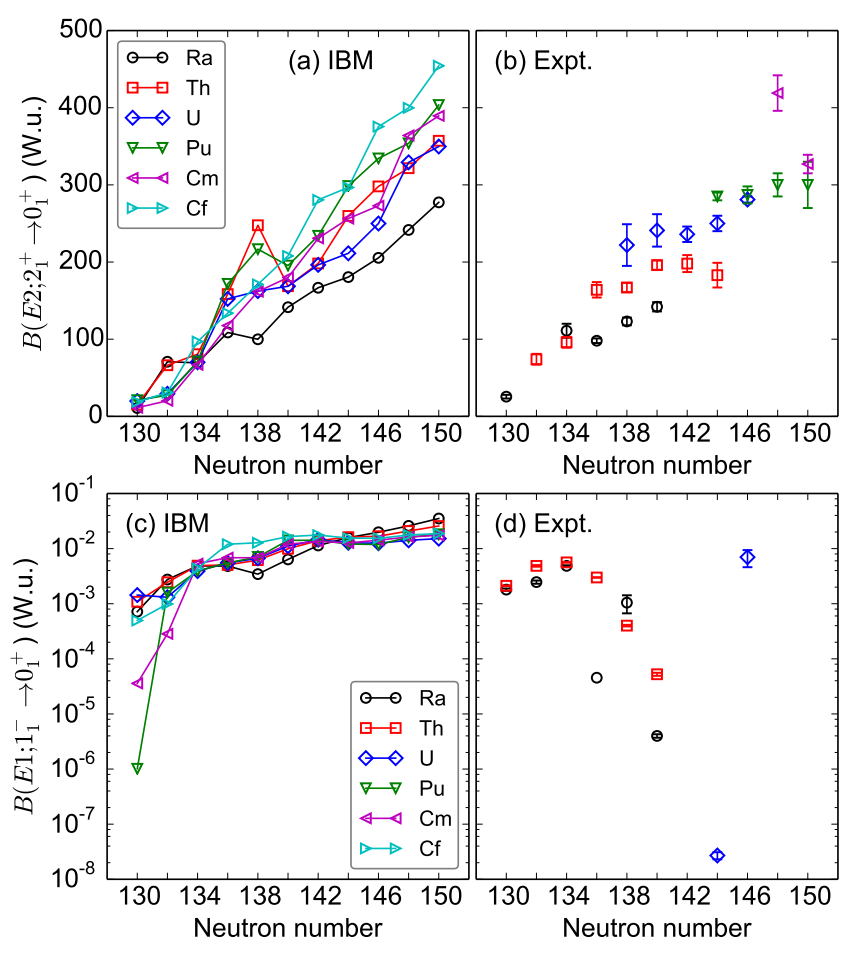

FIG. 10. Evolution of the theoretical and experimental $B\left(E 2 ; 2_{1}^{+} \rightarrow 0_{1}^{+}\right)$(panels $(\mathrm{a})$ and $\left.(\mathrm{b})\right)$ and $B\left(E 1 ; 1_{1}^{-} \rightarrow 0_{1}^{+}\right.$) (panels (c) and (d)) transition strength in Weisskopf units for the Ra, Th, U, Pu, Cm, and Cf isotopes as functions of the neutron number. Theoretical values for the Ra and Th nuclei are taken from Ref. 45. The experimental data are from Refs. 63.

ues. The predicted $B(E 2)$ rates keep increasing with $N$ as the quadrupole collectivity develops, and are in a good agreement with the data. However, the present model is unable to describe, even qualitatively, the empirical $B(E 1)$ rates (panels (c) and (d) of Figs. 10). This is mainly because of the fact that the E1 properties are less collective in nature, while the IBM framework is built on correlated pairs and deals with purely collective states. Another reason is that, within the $s d f$ boson space, the E1 transition operator only contains a term that is proportional to $\left(d^{\dagger} f+f^{\dagger} \tilde{d}\right)^{(1)}$, and this simplified form may not satisfactorily describe the details of the observed $B(E 1)$ systematic. Inclusion of higher-order terms in the E1 operator or taking into account explicitly the dipole $p$ boson with $J=1^{-}$could improve description of the $\mathrm{E} 1$ rates, but these extensions are clearly out of the scope of the present paper.

\section{H. Effective quadrupole and octupole deformations}

We further consider quadrupole and octupole shape invariants 65, 66] calculated in the IBM ground state 

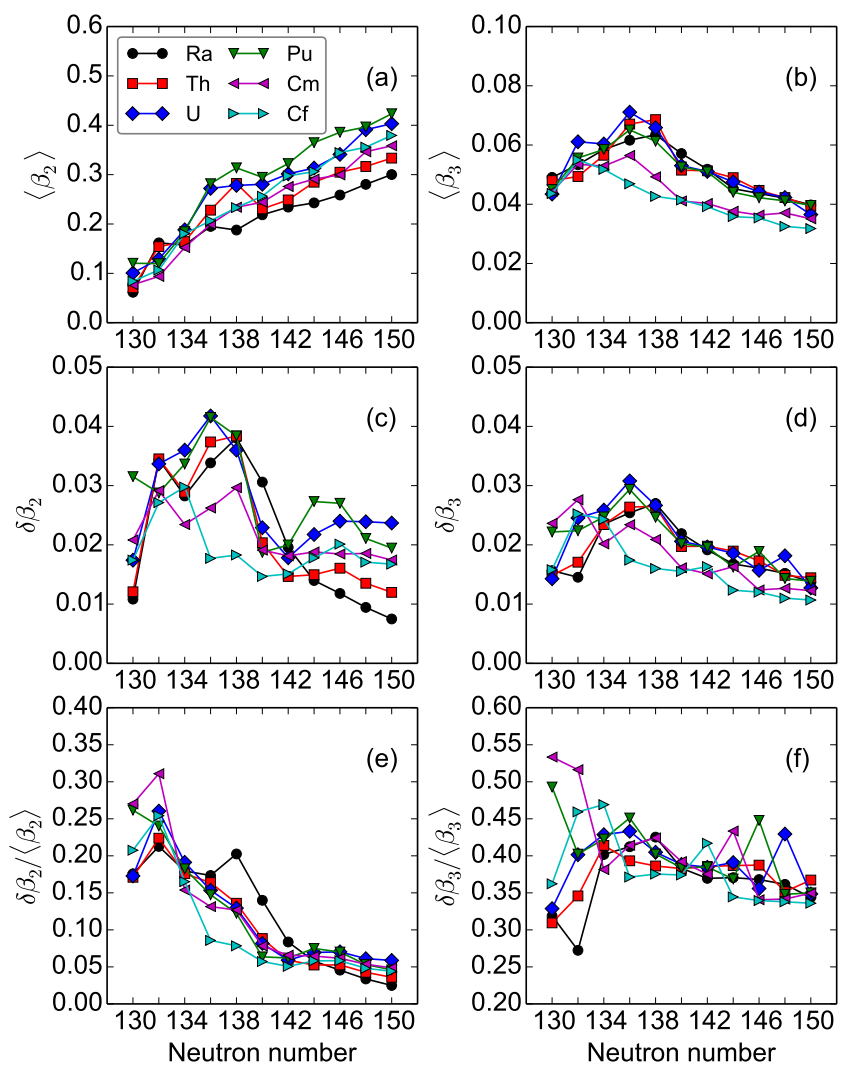

FIG. 11. Effective quadrupole $\left\langle\beta_{2}\right\rangle$ (a) and octupole $\left\langle\beta_{3}\right\rangle$ (b) deformation parameters, variance $\delta \beta_{\lambda}(\mathrm{c}, \mathrm{d})$, and the fluctuations $\delta \beta_{\lambda} /\left\langle\beta_{\lambda}\right\rangle$ (e,f) for ${ }^{218-238} \mathrm{Ra},{ }^{220-240} \mathrm{Th},{ }^{222-242} \mathrm{U}$, ${ }^{224-244} \mathrm{Pu},{ }^{226-246} \mathrm{Cm}$, and ${ }^{228-248} \mathrm{Cf}$, as functions of the neutron number. Values for the $\mathrm{Ra}$ and $\mathrm{Th}$ nuclei have been calculated based on the results from Ref. 45. See the main text for details.

$\left|0_{1}^{+}\right\rangle$:

$$
\begin{gathered}
q_{2}^{(\lambda)}=\sum_{i}(-1)^{J}\left\langle 0_{1}^{+}\left\|\hat{T}^{E \lambda}\right\| J_{i}^{\pi}\right\rangle\left\langle J_{i}^{\pi}\left\|\hat{T}^{E \lambda}\right\| 0_{1}^{+}\right\rangle \\
q_{4}^{(\lambda)}=\sum_{i, j, k}\left\langle 0_{1}^{+}\left\|\hat{T}^{E \lambda}\right\| J_{i}^{\pi}\right\rangle\left\langle J_{i}^{\pi}\left\|\hat{T}^{E \lambda}\right\| 0_{j}^{+}\right\rangle \\
\times\left\langle 0_{j}^{+}\left\|\hat{T}^{E \lambda}\right\| J_{k}^{\pi}\right\rangle\left\langle J_{k}^{\pi}\left\|\hat{T}^{E \lambda}\right\| 0_{1}^{+}\right\rangle
\end{gathered}
$$

where $\left\langle 0^{+}\left\|\hat{T}^{E \lambda}\right\| J^{\pi}\right\rangle$ 's are reduced matrix elements of the $E \lambda$ transition operators, and $J^{\pi}=2^{+}$and $3^{-}$for $\lambda=$ 2 and 3, respectively. The sums in Eqs. (9) and (10) include up to 10 lowest $0^{+}, 2^{+}$, and $3^{-}$states. We can define the effective quadrupole and octupole deformation parameters

$$
\left\langle\beta_{\lambda}\right\rangle=\sqrt{\left\langle\beta_{\lambda}^{2}\right\rangle}
$$

and the variance

$$
\delta \beta_{\lambda}=\sqrt{\left\langle\beta_{\lambda}^{4}\right\rangle-\left\langle\beta_{\lambda}^{2}\right\rangle^{2}} / 2\left\langle\beta_{\lambda}\right\rangle
$$

where $\left\langle\beta_{\lambda}^{2 m}\right\rangle=\left(4 \pi /\left(3 e Z R_{0}^{\lambda}\right)\right)^{2 m} q_{2 m}^{(\lambda)}(m=1,2)$.

In Fig. 11 we display the above mentioned quantities as functions of $N$ : in panels (a) and (b) we show $\left\langle\beta_{\lambda}\right\rangle$ for $\lambda=2$ and 3, respectively. In panels (c) and (d) the $\delta \beta_{\lambda}$ are displayed. Finally, the fluctuations $\delta \beta_{\lambda} /\left\langle\beta_{\lambda}\right\rangle$ are presented in panels (e) and (f). The effective quadrupole deformation $\left\langle\beta_{2}\right\rangle$ increases monotonously with $N$ as the quadrupole collectivity develops. Behavior of the effective octupole deformation parameter $\left\langle\beta_{3}\right\rangle$ for each isotopic chain is characterized by a parabolic trend with a marked peak at $N \approx 136$. The behavior of these quantities with neutron number is in agreement with the one of the intrinsic deformation parameters shown in Fig. 3. The variance for both the quadrupole and octupole deformations is large from $N \approx 132$ to $N \approx 140$. In such transitional regions, potentials are soft in both $\beta_{2}$ and $\beta_{3}$ (cf. Figs. 1 and 2), and large shape fluctuations are present. This is confirmed by rapid changes of the quantities $\delta \beta_{2} /\left\langle\beta_{2}\right\rangle$ and $\delta \beta_{3} /\left\langle\beta_{3}\right\rangle$ between $N \approx 132$ and $N \approx 138$ (cf. panels (e) and (f) of Fig. 11).

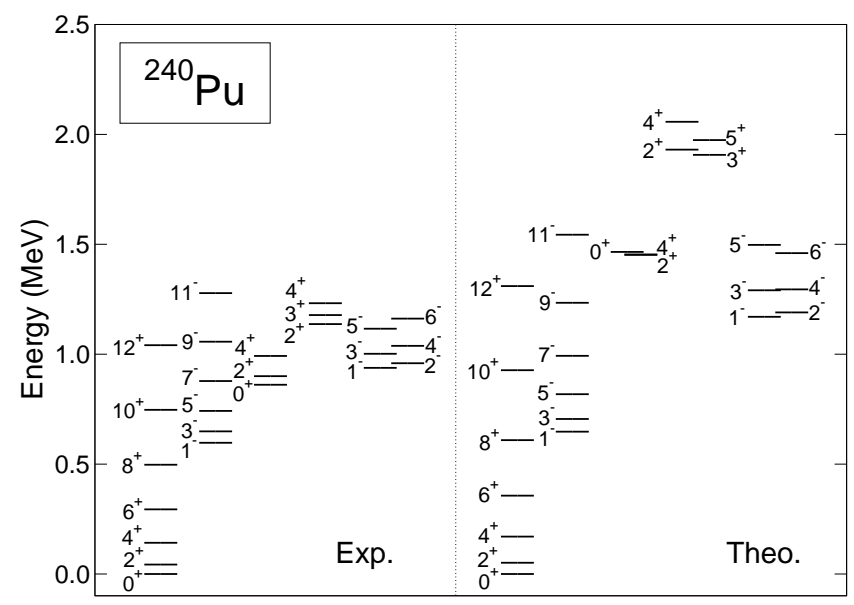

FIG. 12. Comparison of theoretical and experimental lowenergy level schemes of ${ }^{240} \mathrm{Pu}$.

\section{Detailed level scheme of ${ }^{240} \mathbf{P u}$}

Finally, we examine the ability of the present approach to describe detailed spectroscopy in individual nuclei. As an illustrative example, in Fig. 12 we compare theoretical and experimental low-energy level schemes of ${ }^{240} \mathrm{Pu}$. A good agreement between theory and experiment is observed in the lowest-lying members of the positive- and negative-parity rotational bands, as well as in the band built on the $1_{2}^{-}$state. The model, however, considerably overestimates the band-head energies of the excited $K^{\pi}=0^{+}$band built on the $0_{2}^{+}$state and the $\gamma$-vibrational $\left(K^{\pi}=2^{+}\right)$band on top of $2_{3}^{+}$. The discrepancies in the $K^{\pi}=0^{+}$and $2^{+}$bands are most likely related to the fact that the underlying SCMF-PESs have too steep poten- 
tials to be reproduced by the IBM ones. The description of the $\gamma$-band levels could be partly improved by explicitly taking into account the triaxial degree of freedom in the present calculation. It has been shown 67] that a specific form of three-body boson term plays an important role to lower the $\gamma$ band. As we have seen in panels (c) and (d) of Fig. 7, the IBM wave functions of the $0_{2}^{+}$ state and the band built on it for most of the considered actinide nuclei are mainly based on two-octupole-boson configurations. However, the fact that the $0_{2}^{+}$energy is overestimated may indicate a need for including additional building blocks in the IBM framework to improve the agreement with experimental results. For instance, inclusion of the dynamical pairing degree of freedom in the IBM model space has been shown 68 to have a significant impact on the description of the excited $0^{+}$states.

We note that, except for the heavier isotopes $\mathrm{Cm}$ and Cf, a plenty of spectroscopic data is available for the neighboring $\mathrm{U}$ and $\mathrm{Pu}$ nuclei as well. We have then carried out similar analyses, and confirmed that the level of agreement between the predicted and experimental energy levels with both parities for these nuclei is similar to ${ }^{240} \mathrm{Pu}$, while the same problem as seen in ${ }^{240} \mathrm{Pu}$ is commonly observed, that is, both the excited $0^{+}$and $2^{+}$ bands are considerably overestimated within the mapped IBM framework.

\section{SUMMARY}

Octupole shapes and collective excitations in the actinide nuclei $\mathrm{Ra}, \mathrm{Th}, \mathrm{U}, \mathrm{Pu}, \mathrm{Cm}$, and $\mathrm{Cf}$ with neutron number $130 \leqslant N \leqslant 150$ have been investigated by using the EDF-based IBM framework. Axiallysymmetric quadrupole $\beta_{2}$ and octupole $\beta_{3}$ SCMF-PESs, obtained from constrained HFB calculations based on the Gogny-D1M EDF, have been used to determine the $s d f$-IBM Hamiltonian. Diagonalization of the mapped Hamiltonian produces excitation spectra and transition strengths. The Gogny-D1M SCMF-PESs have suggested transitions from nearly spherical $(N \approx 130)$ to stable octupole-deformed $(N \approx 134)$ and to octupole-soft $(N \approx 138)$ shapes along the considered $\mathrm{U}, \mathrm{Pu}, \mathrm{Cm}$, and Cf isotopic chains. Consistently with the empirical tendency, the calculated negative-parity yrast states show a parabolic trend centered at $N \approx 136$, and the $B\left(E 3 ; 3_{1}^{-} \rightarrow 0_{1}^{+}\right)$transition rates are predicted to take maximal values around $N \approx 136$, where the SCMF-PESs exhibit the most pronounced octupole minima. The rotational bands of a number of nuclei exhibit the alternatingparity pattern associated with a rigid octupole shape (cf. Fig. 8). The effective $\beta_{2}$ and $\beta_{3}$ deformations and their variance suggest large shape fluctuations near $N=134$. All the spectroscopic properties obtained from the mapped $s d f$-IBM Hamiltonian exhibit tenden- cies that correlate with the variation of the Gogny-D1M SCMF-PESs, and consistently suggest the onset of stable octupole deformation around $N=136$ and that the transitions between octupole-deformed and octupole-soft shapes occur systematically in the actinide region. The spectroscopic results discussed in this paper also agree well, at least qualitatively, with the recent EDF-based spectroscopic calculations [34, 38] that covered the same region of nuclei as the one studied here.

Even though our model allows for a detailed and economic description of octupole-related spectroscopic properties, the current implementation of the model is not able to describe quantitatively the spectra of non-yrast states. For instance, the model has overestimated considerably the excitation energies of the $0_{2}^{+}$states and the $\gamma\left(K=2^{+}\right)$band (cf. Fig. 12 ). This implies the necessity of including those building blocks that are beyond the considered IBM framework, e.g., dynamical pairing and triaxial degrees of freedom (i.e., higher-order terms in the IBM Hamiltonian). Since these new building blocks have negligible contributions to the yrast states with both parities, they would not alter the conclusion of the present work, that is, the stable octupole shape occurs around $N \approx 134$ and octupole softness emerges around $N \approx 138$ in actinide nuclei. Such extensions of the model will be required for a complete spectroscopic study that involves an accurate description of excitation energies and transition rates of non-yrast states. Another interesting topic is to extend the analysis to odd-mass actinides. Work along these lines is in progress, and will be reported in forthcoming articles.

\section{ACKNOWLEDGMENTS}

This work has been supported by the Tenure Track Pilot Programme of the Croatian Science Foundation and the École Polytechnique Fédérale de Lausanne, and the Project TTP-2018-07-3554 Exotic Nuclear Structure and Dynamics, with funds of the Croatian-Swiss Research Programme. The work of LMR was supported by Spanish Ministry of Economy and Competitiveness (MINECO) Grant No. PGC2018-094583B-I00. This work has been partially supported by the Ministerio de Ciencia e Innovación (Spain) under projects number PID2019-104002GB-C21, by the Consejería de Economía, Conocimiento, Empresas y Universidad de la Junta de Andalucía (Spain) under Group FQM-370, by the European Regional Development Fund (ERDF), ref. SOMM17/6105/UGR, and by the European Commission, ref. H2020-INFRAIA-2014-2015 (ENSAR2). Resources supporting this work were provided by the CEAFMC and the Universidad de Huelva High Performance Computer (HPC@UHU) funded by ERDF/MINECO project UNHU-15CE-2848. 
[1] P. A. Butler and W. Nazarewicz, Rev. Mod. Phys. 68, 349 (1996)

[2] P. A. Butler, J. Phys. G: Nucl. Part. Phys. 43, 073002 (2016)

[3] L. P. Gaffney, P. A. Butler, M. Scheck, A. B. Hayes, F. Wenander, M. Albers, B. Bastin, C. Bauer, A. Blazhev, S. Bönig, N. Bree, J. Cederkäll, T. Chupp, D. Cline, T. E. Cocolios, T. Davinson, H. D. Witte, J. Diriken, T. Grahn, A. Herzan, M. Huyse, D. G. Jenkins, D. T. Joss, N. Kesteloot, J. Konki, M. Kowalczyk, T. Kröll, E. Kwan, R. Lutter, K. Moschner, P. Napiorkowski, J. Pakarinen, M. Pfeiffer, D. Radeck, P. Reiter, K. Reynders, S. V. Rigby, L. M. Robledo, M. Rudigier, S. Sambi, M. Seidlitz, B. Siebeck, T. Stora, P. Thoele, P. V. Duppen, M. J. Vermeulen, M. von Schmid, D. Voulot, N. Warr, K. Wimmer, K. WrzosekLipska, C. Y. Wu, and M. Zielinska, Nature (London) 497, 199 (2013)

[4] P. A. Butler, L. P. Gaffney, P. Spagnoletti, K. Abrahams, M. Bowry, J. Cederkäll, G. de Angelis, H. De Witte, P. E. Garrett, A. Goldkuhle, C. Henrich, A. Illana, K. Johnston, D. T. Joss, J. M. Keatings, N. A. Kelly, M. Komorowska, J. Konki, T. Kröll, M. Lozano, B. S. Nara Singh, D. O'Donnell, J. Ojala, R. D. Page, L. G. Pedersen, C. Raison, P. Reiter, J. A. Rodriguez, D. Rosiak, S. Rothe, M. Scheck, M. Seidlitz, T. M. Shneidman, B. Siebeck, J. Sinclair, J. F. Smith, M. Stryjczyk, P. Van Duppen, S. Vinals, V. Virtanen, N. Warr, K. Wrzosek-Lipska, and M. Zielińska, Phys. Rev. Lett. 124, 042503 (2020)

[5] M. M. R. Chishti, D. O'Donnell, G. Battaglia, M. Bowry, D. A. Jaroszynski, B. S. N. Singh, M. Scheck, P. Spagnoletti, and J. F. Smith, Nat. Phys. 16, 853 (2020)

[6] B. Bucher, S. Zhu, C. Y. Wu, R. V. F. Janssens, D. Cline, A. B. Hayes, M. Albers, A. D. Ayangeakaa, P. A. Butler, C. M. Campbell, M. P. Carpenter, C. J. Chiara, J. A. Clark, H. L. Crawford, M. Cromaz, H. M. David, C. Dickerson, E. T. Gregor, J. Harker, C. R. Hoffman, B. P. Kay, F. G. Kondev, A. Korichi, T. Lauritsen, A. O. Macchiavelli, R. C. Pardo, A. Richard, M. A. Riley, G. Savard, M. Scheck, D. Seweryniak, M. K. Smith, R. Vondrasek, and A. Wiens, Phys. Rev. Lett. 116, 112503 (2016).

[7] B. Bucher, S. Zhu, C. Y. Wu, R. V. F. Janssens, R. N. Bernard, L. M. Robledo, T. R. Rodríguez, D. Cline, A. B. Hayes, A. D. Ayangeakaa, M. Q. Buckner, C. M. Campbell, M. P. Carpenter, J. A. Clark, H. L. Crawford, H. M. David, C. Dickerson, J. Harker, C. R. Hoffman, B. P. Kay, F. G. Kondev, T. Lauritsen, A. O. Macchiavelli, R. C. Pardo, G. Savard, D. Seweryniak, and R. Vondrasek, Phys. Rev. Lett. 118, 152504 (2017).

[8] W. Nazarewicz, P. Olanders, I. Ragnarsson, J. Dudek, G. A. Leander, P. Möller, and E. Ruchowsa, Nucl. Phys. A 429, 269 (1984).

[9] G. Leander, W. Nazarewicz, P. Olanders, I. Ragnarsson, and J. Dudek, Phys. Lett. B 152, 284 (1985).

[10] P. Möller, R. Bengtsson, B. Carlsson, P. Olivius, T. Ichikawa, H. Sagawa, and A. Iwamoto, At. Dat. Nucl. Dat. Tab. 94, 758 (2008).

[11] S. Marcos, H. Flocard, and P. Heenen, Nucl. Phys. A 410, 125 (1983).

[12] P. Bonche, P. Heenen, H. Flocard, and D. Vautherin,
Phys. Lett. B 175, 387 (1986).

[13] P. Bonche, S. J. Krieger, M. S. Weiss, J. Dobaczewski, H. Flocard, and P.-H. Heenen, Phys. Rev. Lett. 66, 876 (1991)

[14] P.-H. Heenen, J. Skalski, P. Bonche, and H. Flocard, Phys. Rev. C 50, 802 (1994).

[15] L. M. Robledo, J. L. Egido, J. Berger, and M. Girod, Phys. Lett. B 187, 223 (1987).

[16] L. M. Robledo, J. L. Egido, B. Nerlo-Pomorska, and K. Pomorski, Phys. Lett. B 201, 409 (1988)

[17] J. L. Egido and L. M. Robledo, Nucl. Phys. A 518, 475 (1990)

[18] J. L. Egido and L. M. Robledo, Nucl. Phys. A 524, 65 (1991)

[19] J. L. Egido and L. M. Robledo, Nucl. Phys. A 545, 589 (1992)

[20] E. Garrote, J. L. Egido, and L. M. Robledo, Phys. Rev. Lett. 80, 4398 (1998)

[21] E. Garrote, J. L. Egido, and L. M. Robledo, Nucl. Phys. A 654, 723c (1999).

[22] W. Long, J. Meng, N. V. Giai, and S.-G. Zhou, Phys. Rev. C 69, 034319 (2004).

[23] L. M. Robledo, M. Baldo, P. Schuck, and X. Viñas, Phys. Rev. C 81, 034315 (2010).

[24] L. M. Robledo and G. F. Bertsch, Phys. Rev. C 84, $054302(2011)$

[25] J. Erler, K. Langanke, H. P. Loens, G. Martínez-Pinedo, and P.-G. Reinhard, Phys. Rev. C 85, 025802 (2012)

[26] L. M. Robledo and R. R. Rodríguez-Guzmán, Journal of Physics G: Nuclear and Particle Physics 39, 105103 (2012),

[27] R. Rodríguez-Guzmán, L. M. Robledo, and P. Sarriguren, Phys. Rev. C 86, 034336 (2012).

[28] L. M. Robledo and P. A. Butler, Phys. Rev. C 88, 051302 (2013).

[29] L. M. Robledo, J. Phys. G: Nucl. Part. Phys. 42, 055109 (2015).

[30] R. N. Bernard, L. M. Robledo, and T. R. Rodríguez, Phys. Rev. C 93, 061302 (2016).

[31] S. E. Agbemava, A. V. Afanasjev, and P. Ring, Phys. Rev. C 93, 044304 (2016).

[32] S. E. Agbemava and A. V. Afanasjev, Phys. Rev. C 96, 024301 (2017)

[33] Z. Xu and Z.-P. Li, Chin. Phys. C 41, 124107 (2017)

[34] S. Y. Xia, H. Tao, Y. Lu, Z. P. Li, T. Nikšić, and D. Vretenar, Phys. Rev. C 96, 054303 (2017).

[35] S. Ebata and T. Nakatsukasa, Physica Scripta 92, 064005 (2017).

[36] R. Rodríguez-Guzmán, Y. M. Humadi, and L. M. Robledo, Eur. Phys. J. A 56, 43 (2020)

[37] Y. Cao, S. E. Agbemava, A. V. Afanasjev, W. Nazarewicz, and E. Olsen, Phys. Rev. C 102, $024311(2020)$

[38] R. Rodríguez-Guzmán, Y. M. Humadi, and L. M. Robledo, J. Phys. G: Nucl. Part. Phys. 48, 015103 (2020).

[39] J. Engel and F. Iachello, Nucl. Phys. A 472, 61 (1987)

[40] N. V. Zamfir and D. Kusnezov, Phys. Rev. C 63, 054306 (2001)

[41] N. V. Zamfir and D. Kusnezov, Phys. Rev. C 67, 014305 (2003).

[42] K. Nomura, D. Vretenar, and B.-N. Lu, Phys. Rev. C 88, 
$021303(2013)$

[43] K. Nomura, D. Vretenar, T. Nikšić, and B.-N. Lu, Phys. Rev. C 89, 024312 (2014).

[44] K. Nomura, R. Rodríguez-Guzmán, and L. M. Robledo, Phys. Rev. C 92, 014312 (2015).

[45] K. Nomura, R. Rodríguez-Guzmán, Y. M. Humadi, L. M. Robledo, and J. E. García-Ramos, Phys. Rev. C 102, 064326 (2020)

[46] D. Bonatsos, D. Lenis, N. Minkov, D. Petrellis, and P. Yotov, Phys. Rev. C 71, 064309 (2005).

[47] D. Lenis and D. Bonatsos, Phys. Lett. B 633, 474 (2006)

[48] P. G. Bizzeti and A. M. Bizzeti-Sona, Phys. Rev. C 88, 011305 (2013)

[49] T. M. Shneidman, G. G. Adamian, N. V. Antonenko, R. V. Jolos, and W. Scheid, Phys. Lett. B 526, 322 (2002).

[50] T. M. Shneidman, G. G. Adamian, N. V. Antonenko, R. V. Jolos, and W. Scheid, Phys. Rev. C 67, 014313 (2003)

[51] K. Nomura, N. Shimizu, and T. Otsuka, Phys. Rev. Lett. 101, 142501 (2008)

[52] F. Iachello and A. Arima, The interacting boson model (Cambridge University Press, Cambridge, 1987).

[53] T. Nikšić, D. Vretenar, and P. Ring, Phys. Rev. C 78, $034318(2008)$

[54] S. Goriely, S. Hilaire, M. Girod, and S. Péru, Phys. Rev. Lett. 102, 242501 (2009).

[55] P. Ring and P. Schuck, The nuclear many-body problem
(Berlin: Springer-Verlag, 1980).

[56] M. Bender, P.-H. Heenen, and P.-G. Reinhard, Rev. Mod. Phys. 75, 121 (2003).

[57] L. M. Robledo, T. R. Rodríguez, and R. R. RodríguezGuzmán, J. Phys. G: Nucl. Part. Phys. 46, 013001 (2019)

[58] M. Spieker, S. Pascu, D. Bucurescu, T. M. Shneidman, T. Faestermann, R. Hertenberger, H.-F. Wirth, N.-V. Zamfir, and A. Zilges, Phys. Rev. C 97, 064319 (2018).

[59] J. N. Ginocchio and M. W. Kirson, Nucl. Phys. A 350, 31 (1980)

[60] S. Heinze (2008), computer program ARBMODEL (University of Cologne).

[61] J. F. Berger, M. Girod, and D. Gogny, Nucl. Phys. A 428, 23 (1984)

[62] F. Chappert, M. Girod, and S. Hilaire, Phys. Lett. B 668, 420 (2008)

63] Brookhaven National Nuclear Data Center, http://www.nndc.bnl.gov.

[64] T. Kibédi and R. Spear, Atomic Data and Nuclear Data Tables 80, 35 (2002)

[65] D. Cline, Ann. Rev. Nucl. Part. Sci. 36, 683 (1986)

[66] V. Werner, N. Pietralla, P. von Brentano, R. F. Casten, and R. V. Jolos, Phys. Rev. C 61, 021301 (2000).

[67] K. Nomura, N. Shimizu, D. Vretenar, T. Nikšić, and T. Otsuka, Phys. Rev. Lett. 108, 132501 (2012)

[68] K. Nomura, D. Vretenar, Z. P. Li, and J. Xiang, Phys. Rev. C 102, 054313 (2020). 